\title{
Parameter choices for a muon recirculating linear accelerator from 5 to $63 \mathrm{GeV}$
}

\author{
J.S. Berg
}

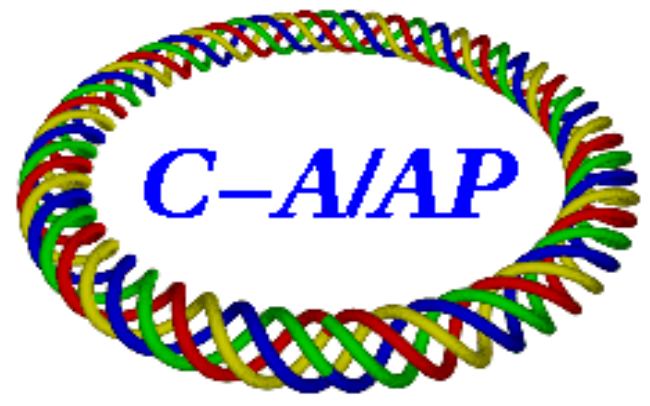

Collider-Accelerator Department Brookhaven National Laboratory Upton, NY 11973

Notice: This document has been authorized by employees of Brookhaven Science Associates, LLC under Contract No. DE-AC02-98CH10886 with the U.S. Department of Energy. The United States Government retains a nonexclusive, paid-up, irrevocable, world-wide license to publish or reproduce the published form of this document, or allow others to do so, for United States Government purposes. 


\title{
Parameter Choices for a Muon Recirculating Linear Accelerator from 5 to 63 GeV
}

\author{
J. Scott Berg* \\ Brookhaven National Laboratory; Building 901A; PO Box 5000; Upton, NY 11973-5000; USA ${ }^{\dagger}$
}

(Dated: June 19, 2014)

\begin{abstract}
A recirculating linear accelerator (RLA) has been proposed to accelerate muons from 5 to $63 \mathrm{GeV}$ for a muon collider. It should be usable both for a Higgs factory and as a stage for a higher energy collider. I first compute constraints on the design due to beam loading. I next find an expression for the longitudinal emittance growth to lowest order in the longitudinal emittance. I then describe a simplified model for the arcs and find an approximate expression for the time of flight dependence on energy in those arcs. I finally use these results to estimate the parameters required for the RLA arcs and the linac phase.
\end{abstract}

TABLE I. Beam and machine parameters [1].

\begin{tabular}{lrrr}
\hline \hline Particles per bunch & $2 \times 10^{12}$ & $4 \times 10^{12}$ & $2 \times 10^{12}$ \\
Longitudinal emittance $(\mathrm{mm})$ & 1.0 & 1.5 & 70 \\
Transverse emittance $(\mu \mathrm{m})$ & 400 & 200 & 25 \\
Initial total energy $(\mathrm{GeV})$ & 5 & 5 & 5 \\
Final total energy $(\mathrm{GeV})$ & 63 & 63 & 63 \\
Maximum emittance growth $(\%)$ & & 10 & \\
Maximum decay loss $(\%)$ & & 11.0 & \\
Physical aperture $(\sigma)$ & & 4.5 & \\
\hline \hline
\end{tabular}

TABLE II. Assumed cavity parameters. $650 \mathrm{MHz}$ values and input power based on [2], $1300 \mathrm{MHz}$ values are based on achieved values, as shown in [3]. Cells per cavity at $325 \mathrm{MHz}$ is based on the LEP2 cavities [4].

\begin{tabular}{lrrrr}
\hline \hline Frequency (MHz) & 325 & 650 & 975 & 1300 \\
\hline Gradient (MV/m) & 20 & 25 & 30 & 35 \\
Maximum cells per cavity & 4 & 5 & 7 & 9 \\
Additional length at each end (cells) & & 1.5 & \\
Maximum input power per cavity (MW) & \multicolumn{4}{c}{1.2} \\
\hline \hline
\end{tabular}

\section{MACHINE PARAMETERS AND GOALS}

I am describing the design of a dogbone geometry [5] recirculating linear accelerator (RLA) that will accelerate a muon beam to $63 \mathrm{GeV}$. It will accelerate for two phases of a Higgs factory, starting with a $5 \mathrm{GeV}$ beam. It will also be re-used for a higher energy collider with substantially larger longitudinal emittance.

The relevant machine parameters and requirements are given in Table I. RF cavity parameters are given in Table II. Requirements on maximum longitudinal emittance growth are based on the energy acceptance of the collider ring [6] in rela-

\footnotetext{
* jsberg@bnl.gov; http://pubweb.bnl.gov/ jsberg/

$\dagger$ This manuscript has been authored by employees of Brookhaven Science Associates, LLC under Contract No. DE-AC02-98CH10886 with the U.S. Department of Energy. The United States Government retains a nonexclusive, paid-up, irrevocable, world-wide license to publish or reproduce the published form of this manuscript, or allow others to do so, for United States Government purposes.
}

TABLE III. $(R / Q) / n_{C}$ for various cavity design.

\begin{tabular}{lr}
\hline \hline Design & $(R / Q) / n_{C}(\Omega)$ \\
\hline Study II, 201.25 MHz, 300 mm aperture [7] & 129 \\
Study II, 201.25 MHz, $460 \mathrm{~mm}$ aperture [7] & 104 \\
SPL, 704 MHz [2] & 114 \\
ILC, $1300 \mathrm{MHz}[8]$ & 115 \\
\hline \hline
\end{tabular}

tion to the RMS energy spread and bunch length for a $70 \mathrm{~mm}$ longitudinal emittance, assuming we want to keep $3 \sigma$, and approximately distributing the allowed growth through the acceleration chain. Transverse emittance growth is limited to the same value. The physical aperture is chosen to keep loss from truncation of a 6-D Gaussian ellipsoid in phase space below $1 \%$.

\section{BEAM LOADING}

The stored energy in a cavity with RF frequency $\omega /(2 \pi)$ and where the peak energy gain of a particle with charge $q$ is $q V$ is

$$
\frac{V^{2}}{\omega(R / Q)}
$$

where $R / Q$ is a parameter related only to the cavity shape (and is indepenent of RF frequency if all the cavity dimensions are scaled inversely with the RF frequency), and is approximately proportional to the number of cells in the cavity.

The energy lost from the cavity when a particle passes through with a phase $\phi$ (with zero being the crest) is $e N V \cos \phi$, where $N$ is the number of particles in the bunch.

In the RLA, we make $n$ passes through the linac with $N$ particles of each signs. Assuming we have the same RF phase on each pass, the fractional reduction in the cavity voltage by the end of the acceleration cycle is cycle is

$$
\frac{\Delta V}{V}=\frac{e n N \omega(R / Q) \cos \phi}{V}
$$

The RF gradient $G$ is defined such that $V=n_{C} G \pi c / \omega$, where $n_{C}$ is the number of cavity cells. Then the fractional reduction in the cavity voltage is

$$
\frac{\Delta V}{V}=\frac{e n N \omega^{2}\left[(R / Q) / n_{C}\right] \cos \phi}{\pi G c}
$$


TABLE IV. Fractional voltage reduction, computed for $\phi=0$.

\begin{tabular}{lrrrr}
\hline \hline Particles & $\begin{array}{r}2 \times 10^{12} \\
\text { Frequency }\end{array}$ & $\begin{array}{c}4 \times 10^{12} \\
325 \mathrm{MHz}\end{array}$ & $\begin{array}{r}2 \times 10^{12} \\
325 \mathrm{MHz}\end{array}$ & $\begin{array}{r}4 \times 10^{12} \\
650 \mathrm{MHz}\end{array}$ \\
\hline Passes & \multicolumn{4}{c}{ Relative reduction $(\%)$} \\
\hline 3 & 2 & 5 & 8 & 16 \\
5 & 4 & 8 & 13 & 26 \\
7 & 6 & 11 & 18 & 36 \\
9 & 7 & 15 & 23 & 47 \\
\hline \hline
\end{tabular}

The geometric parameter $(R / Q) / n_{C}$ for several existing cavities is given in Table III. I will use $114 \Omega$ in subsequent calculations, since it is an intermediate value amongst these designs. Table IV gives $\Delta V / V$ for varous numbers of passes.

The fractional recution in the stored energy is just

$$
\frac{\Delta U}{U}=2 \frac{\Delta V}{V}-\left(\frac{\Delta V}{V}\right)^{2}
$$

To compensate for beam loading, the installed voltage must be increased by a factor of

$$
\frac{1}{1-\frac{1}{2} \frac{\Delta V}{V}}
$$

Based on empirical observations, I find that one can extract about $50 \%$ of the stored energy in a cavity and still have reasonably stable operation. Thus, for $4 \times 10^{12}$ particles per charge, 9 passes for $325 \mathrm{MHz} \mathrm{RF}$ and 3 passes for $650 \mathrm{MHz}$ are safe. 5 passes at $650 \mathrm{MHz}$ meets the criterion, but just barely. More than 9 passes in a dogbone RLA will likely produce severe difficulties with the switchyard.

Comparing a 9-pass $325 \mathrm{MHz}$ RLA to a 3-pass $650 \mathrm{MHz}$ RLA, the linac costs are similar, assuming the linac per unit energy gain cost goes as the inverse square of the RF frequency. The arc costs for the 9-pass case should be around four times higher when compared to the 3-pass case. Thus, the 3-pass, $650 \mathrm{MHz}$ RLA should be less expansive than the 9-pass $325 \mathrm{MHz}$ RLA.

In a dogbone RLA, the bunches of different signs follow each other down the linac. Thus, one charge will gain less energy than the other charge, if both charges come through at the same phase. For the case of $4 \times 10^{12}$ muons per bunch and $650 \mathrm{MHz}$ RF, that difference will be about $2.6 \%$. To compensate, the later bunch could be accelerated at a different phase than the earlier one.

From the point of view or beam dynamics and collective effects, it is probably preferable to use a racetrack geometry rather than a dogbone. Not only are both signs treated equally, but the synchrotron phase advance is distributed more evenly (and one may have the opportunity to increase the synchrotron phase advance). However, this will increase the number of beamlines for a given number of linac passes. At $650 \mathrm{MHz}$, for instance, three passes through a dogbone RLA linac would require switchyards with four beamlines at each end (including injection/extraction lines), two pairs going in opposite directions. For a racetrack geometry, a solution with four linac
TABLE V. Droplet structure

\begin{tabular}{rrr}
\hline \hline Cells & Angle & Length \\
\hline 2 & $-\theta_{m} / 2$ & $L_{m}$ \\
$n_{o}$ & $-\theta$ & $L$ \\
2 & 0 & $L$ \\
$n_{i}$ & $\theta$ & $L$ \\
2 & 0 & $L$ \\
$n_{o}$ & $-\theta$ & $L$ \\
2 & $-\theta_{m} / 2$ & $L_{m}$ \\
\hline \hline
\end{tabular}

passes through one linac and three through the other would give four beamlines at each end, but they are all heading in the same direction, so from the point of view of magnet density, one has essentially twice the beamline density as the dogbone solution. Nonetheless, I will only deal with the dogbone geometry for the remainder of this paper.

\section{DROPLET ARC DESIGN I}

To study the longitudinal dynamics, it is helpful to have an approximate design for the droplet arcs in the dogbone RLA. Based on the design in [9], the droplet arc will have the following structure:

- Matching cells, which match zero dispersion at the linac to the dispersion in the outward bending cells. There are two of these cells.

- Outward bending cells.

- Dispersion flip cells, which flip the dispersion from the outward bending cells to the inward bending cells. There are two such cells.

- Inward bending cells.

- Dispersion flip cells (2).

- Outward bending cells.

- Matching cells (2).

All cells have a $90^{\circ}$ betatron phase advance. Except for the matching cells, all the cells have the same length $L$. The inward and outward bending cells have bending angles of the same magnitude, $\theta$. The matching cells have a length close to $L$, and a bending angle close to $\theta / 2$. The dispersion flip cells do not bend. Table $\mathrm{V}$ summarizes these parameters.

In a small angle approximation, dispersion matching requires that

$$
L \theta=L_{m} \theta_{m}
$$

Geometric constraints lead to the equations

$$
\left(n_{i}-2 n_{o}\right) \theta-2 \theta_{m}=\pi
$$




$$
\begin{aligned}
& 2 L_{m} \theta\left(1-\cos \theta_{m}\right)+L \theta_{m}\left[\cos \theta_{m}-\cos \left(n_{o} \theta+\theta_{m}\right)\right] \\
& +2 L \theta \theta_{m} \sin \left(n_{o} \theta+\theta_{m}\right)=L \theta_{m} \sin \left(n_{i} \theta / 2\right)
\end{aligned}
$$

Defining $r=\theta_{m} / \theta=L / L_{m}$, the above equations give a single equation. Ignoring the constraint that $n_{i}$ must be an integer, we can write

$$
\left(2-r^{2}\right) \cos (r \theta)+2 r^{2} \cos \left[\left(n_{o}+r\right) \theta\right]-2 r^{2} \theta \sin \left[\left(n_{o}+r\right) \theta\right]=2
$$

There are two free variables for a given $n_{o}: r$ and $\theta$. My first step is to set $r=1$ and solve this equation for $\theta$. The equation that I solve is therefore

$$
f(\theta)=\cos \theta+2 \cos \left[\left(n_{o}+1\right) \theta\right]-2 \theta \sin \left[\left(n_{o}+1\right) \theta\right]-2=0
$$

For Newton's method, we would like to have the derivative of $f$,

$$
\begin{aligned}
f^{\prime}(\theta)=-\sin \theta-2\left(n_{o}+2\right) \sin \left[\left(n_{o}+1\right) \theta\right] \\
-2 \theta\left(n_{o}+1\right) \cos \left[\left(n_{o}+1\right) \theta\right]
\end{aligned}
$$

For small angles,

$$
f(\theta) \approx 1-\theta^{2}\left(n_{o}^{2}+4 n_{o}+7 / 2\right)
$$

and therefore an initial guess for $\theta$ is

$$
\theta \approx\left(n_{o}^{2}+4 n_{o}+7 / 2\right)^{-1 / 2}
$$

Starting with this value for $\theta$, compute $n_{i}$ :

$$
n_{i}=\pi / \theta+2\left(n_{o}+1\right)
$$

The interesting result is that $n_{i}$ is very close to being $5 n_{o}+8$. I set $n_{i}=5 n_{o}+8$. Then

$$
\theta=\frac{\pi}{3 n_{o}+8-2 r}
$$

Then we have to solve

$$
\begin{array}{r}
g(r)=\left(2-r^{2}\right) \cos \frac{\pi r}{3 n_{o}+8-2 r}+2 r^{2} \cos \frac{\pi\left(n_{o}+r\right)}{3 n_{o}+8-2 r} \\
-\frac{2 r^{2} \pi}{3 n_{o}+8-2 r} \sin \frac{\pi\left(n_{o}+r\right)}{3 n_{o}+8-2 r}-2=0
\end{array}
$$

for $r$. The derivative of $g(r)$ is

$$
\begin{array}{r}
g^{\prime}(r)=-2 r \cos \frac{\pi r}{3 n_{o}+8-2 r} \\
-\frac{\pi\left(2-r^{2}\right)\left(3 n_{o}+8\right)}{\left(3 n_{o}+8-2 r\right)^{2}} \sin \frac{\pi r}{3 n_{o}+8-2 r} \\
\quad+4 r \cos \frac{\pi\left(n_{o}+r\right)}{3 n_{o}+8-2 r} \\
-\frac{2 \pi r^{2}\left(5 n_{o}+8\right)}{\left(3 n_{o}+8-2 r\right)^{2}} \sin \frac{\pi\left(n_{o}+r\right)}{3 n_{o}+8-2 r} \\
-\frac{4 \pi r\left(3 n_{o}+8-r\right)}{\left(3 n_{o}+8-2 r\right)^{2}} \sin \frac{\pi\left(n_{o}+r\right)}{3 n_{o}+8-2 r} \\
\quad-\frac{2 \pi^{2} r^{2}\left(5 n_{o}+8\right)}{\left(3 n_{o}+8-2 r\right)^{3}} \cos \frac{\pi\left(n_{o}+r\right)}{3 n_{o}+8-2 r}
\end{array}
$$

The solution to $g(r)=0$ is well approximated by

$$
r=1+\frac{1}{5}\left(\frac{\pi}{3 n_{o}+6}\right)^{2}+O\left[\left(\frac{\pi}{3 n_{o}+6}\right)^{3}\right] .
$$

Even for $n_{o}=0$, the difference of $r$ from 1 is around 0.05 , and that difference decreases quickly as $n_{o}$ increases.

\section{LONGITUDINAL DYNAMICS}

\section{A. Droplet Arc}

I will model the arcs as having a linear time dependence on energy, $T_{0}+T_{1} \Delta$, where $\Delta$ is the difference of the energy from some reference energy. In addition to the approximations I describe above, I make the following approximations:

- I use thin lens quadrupoles.

- The spaces between quadrupoles are dipoles.

- Bend angles are small.

- I ignore focusing from the dipoles.

The resulting time depencence on energy for the full arc is given by

$$
\begin{aligned}
& T_{1}=\frac{7 E^{2}\left[L_{m} \theta_{m}^{2}+L \theta^{2}\left(2 n_{o}+n_{i}\right)\right]}{16 c^{4} p^{3}} \\
&-\frac{m^{2}\left[4 L_{m}+\left(4+2 n_{o}+n_{i}\right) L\right]}{p^{3}}
\end{aligned}
$$

For an average dipole field $B$, we have that $L=p \theta /(q B)$, and thus

$$
\begin{aligned}
T_{1}=\frac{7 E^{2}\left[\theta_{m}^{3}+\theta^{3}\left(2 n_{o}+n_{i}\right)\right]}{16 q B c^{4} p^{2}} & \\
& -\frac{m^{2}\left[4 \theta_{m}+\left(4+2 n_{o}+n_{i}\right) \theta\right]}{q B p^{2}}
\end{aligned}
$$

For highly relativistic particles, this is

$$
T_{1}=\frac{7\left[\theta_{m}^{3}+\theta^{3}\left(2 n_{o}+n_{i}\right)\right]}{16 q B c^{2}}
$$

From the above calculations, a good estimates for $\theta$ and $\theta_{m}$ are

$$
\theta_{m} \approx \theta \approx \frac{\pi}{3\left(n_{o}+2\right)}
$$

Then my final approximation for $T_{1}$ is

$$
T_{1} \approx \frac{7 \pi^{2}\left(7 n_{o}+9\right) L}{144\left(n_{o}+2\right)^{2} p c^{2}} \approx \frac{7 \pi^{3}\left(7 n_{o}+9\right)}{432\left(n_{o}+2\right)^{3} q B c^{2}}
$$




\section{B. Nonlinear Dynamics}

In my model, the time of flight depends linear on energy as above. The RF contribution is treated as a single kick. All arcs will have the same $T_{1}$, which can be achieved by scaling all the lengths linearly in the arc design momentum. Using the notation from [10], the map can be written as

$$
e^{-: H_{20}:} e^{-: H_{n 0}:}
$$

where

$$
\begin{aligned}
& e^{-: H_{20}:}=e^{: T_{1} \Delta^{2} / 4:} e^{:(V \omega \sin \phi) \tau^{2} / 2:} e^{: T_{1} \Delta^{2} / 4:} \\
& e^{-: H_{n 0}:}=e^{-: T_{1} \Delta^{2} / 4} e^{-: H_{R F, n}:} e^{: T_{1} \Delta^{2} / 4:}
\end{aligned}
$$

and

$$
H_{R F, n}=\frac{V}{\omega} \sin (\omega \tau+\phi)-\frac{V}{\omega} \sin \phi-V \tau \cos \phi+\frac{1}{2}(V \omega \sin \phi) \tau^{2}
$$

I will estimate the emittance growth arising from the nonnlinear portion of the Hamiltonian. The incoming distribution is matched to the linear part of the Hamiltonian. Define a linear transformation matrix $A_{2}$ such that if

$$
\left[\begin{array}{l}
\Delta \\
\tau
\end{array}\right]=A_{2}\left[\begin{array}{l}
q \\
p
\end{array}\right]
$$

then the map in these transformed coordinates is

$$
e^{-\mu: q_{1}^{2}+p_{1}^{2}: / 2} e^{-: H_{n 1}:}
$$

The distribution function for the incoming distribution will depend only on $J_{1}=\left(q_{1}^{2}+p_{1}^{2}\right) / 2$. We can approximately normalize the full nonlinear Hamiltonian to find phase space coordinates $\left(q_{2}, p_{2}\right)$ such that $J_{2}=\left(q_{2}^{2}+p_{2}^{2}\right) / 2$ is approximately constant. The emittance that the beam will eventually filament to is the expectation value of $\boldsymbol{J}_{2}$ for the incoming distribution.

I approximate the nonlinear transformation from $\left(q_{1}, p_{1}\right)$ to $\left(q_{2}, p_{2}\right)$ by

$$
e^{: A_{3}:} e^{: A_{4}:}
$$

where $A_{k}$ is a homogeneous polynomial of order $k$. In fact, it is only necessary to comptue $A_{3}$, since $A_{4}$ will not contribute to the emittance growth (though the calculation will require computing fourth order terms). If $A_{3}$ is

$$
A_{30} q^{3}+A_{31} q^{2} p+A_{32} q p^{2}+A_{33} p^{3},
$$

then the emittance growth, assuming complete filamentation, is

$$
\begin{aligned}
{\left[\frac{27}{4}\left(A_{30}^{2}+A_{33}^{2}\right)+\frac{15}{4}\right.} & \left(A_{31}^{2}+A_{32}^{2}\right) \\
& \left.-\frac{9}{2}\left(A_{31} A_{33}+A_{30} A_{32}\right)\right]\left\langle J^{2}\right\rangle
\end{aligned}
$$

For a uniform phase space density (waterbag) distribution, $\left\langle J^{2}\right\rangle=4 \epsilon^{2} / 3$; for a Gaussian distribution, $\left\langle J^{2}\right\rangle=2 \epsilon^{2}$.
For our system, the phase advance $\mu$ is given by the equation

$$
2 \sin \frac{\mu}{2}=-\sqrt{T_{1} V \omega \sin \phi}
$$

and the transformation matrix $A_{2}$ is

$$
\left[\begin{array}{cc}
\sqrt{\frac{V \omega \sin \phi}{T_{1}}} \sec \frac{\mu}{2} & 0 \\
0 & \sqrt{\frac{T_{1}}{V \omega \sin \phi}} \cos \frac{\mu}{2}
\end{array}\right]
$$

I write

$$
H_{n 0}=\sum_{k=3}^{\infty} H_{k 0}
$$

where $H_{k 0}$ is a homogeneous polynomial in $\tau$ and $\Delta$ of degree $k$. The only term I will need is $H_{30}$, which is

$$
H_{30}=-\frac{1}{6}\left(V \omega^{2} \cos \phi\right) \tau^{3}
$$

Similarly, I write

$$
H_{n 1}=\sum_{k=3}^{\infty} H_{k 1}
$$

and

$$
\begin{aligned}
H_{31}=-\frac{1}{6} V \omega^{2}\left(\frac{T_{1}}{2}\right)^{3 / 2} \cos \phi & \\
& \left(p \cot ^{1 / 2} \frac{-\mu}{2}-q \tan ^{1 / 2} \frac{-\mu}{2}\right)^{3}
\end{aligned}
$$

The $A_{3}$ that generates the transformation from a new set of phase space variables $\left(q_{2}, p_{2}\right)$ to $\left(q_{1}, p_{1}\right)$ can be found by solving the equation

$$
\left[A_{3}, H_{21}\right]+H_{31}=0 \text {. }
$$

The result is

$$
\begin{aligned}
& A_{3}=\frac{1}{6} \frac{V \omega^{2}}{\mu}\left(\frac{T_{1}}{2}\right)^{3 / 2} \cos \phi[ \\
& q^{3}\left(\frac{2}{3} \cot ^{3 / 2} \frac{-\mu}{2}-\cot ^{1 / 2} \frac{-\mu}{2}\right)+q^{2} p \tan ^{3 / 2} \frac{-\mu}{2} \\
&\left.+q p^{2} \cot ^{3 / 2} \frac{-\mu}{2}+p^{3}\left(\frac{2}{3} \tan ^{3 / 2} \frac{-\mu}{2}-\tan ^{1 / 2} \frac{-\mu}{2}\right)\right]
\end{aligned}
$$

The emittance growth is therefore

$$
\Delta \epsilon=\frac{5}{48} \frac{V^{2} \omega^{4} T_{1}^{3} \cos ^{2} \phi}{\mu^{2} \sin ^{3} \mu}\left\langle J^{2}\right\rangle
$$

We desire a particular energy gain per linac pass, which I will call $U=V \cos \phi$. Therefore the emittance growth can be rewritten as

$$
\Delta \epsilon=-\frac{5}{48} \frac{U^{2} \omega^{4} T_{1}^{3}}{\mu^{2} \sin ^{3} \mu}\left\langle J^{2}\right\rangle
$$


TABLE VI. Parameters required to limit longitudinal emittance growth.

\begin{tabular}{lrrrr}
\hline \hline$\epsilon(\mathrm{mm})$ & 1.5 & 1.5 & 70 & 70 \\
$\omega / 2 \pi(\mathrm{MHz})$ & 325 & 650 & 325 & 650 \\
Linac passes & 9 & 3 & 9 & 3 \\
\hline$T_{1}(\mathrm{ps} / \mathrm{GeV})$ & 1567 & 299 & 435 & 83 \\
$\phi(\mathrm{deg})$ & 7 & 6 & 25 & 22 \\
$\sigma_{E}(\mathrm{MeV})$ & 22 & 50 & 283 & 647 \\
\hline \hline
\end{tabular}

with

$$
2 \sin \frac{\mu}{2}=-\sqrt{T_{1} U \omega \tan \phi}
$$

Therefore, to determine the phase of the RF that will satisfy the emittance growth constraints, we solve the equation

$$
\mu^{2} \sin ^{3} \mu=-\frac{5}{48} \frac{U^{2} \omega^{4} T_{1}^{3} \epsilon}{\Delta \epsilon / \epsilon} \frac{\left\langle J^{2}\right\rangle}{\epsilon^{2}}
$$

The function on the left hand side reaches a maximum when $\mu \approx 1.91 \equiv \mu_{0}$, and its value there is approximately 3.06. Therefore, when the magnitude of the right hand side is greater than 3.06, the emittance growth requirement cannot be met. Note that this synchrotron phase advance is large enough that there may be issues with the synchrotron bucket breaking up.

The next step is to choose parameters that meet the emittance growth requiremetns. This can be done by solving for $T_{1}$, assuming that $\mu=-\mu_{0}$ :

$$
T_{1}=\left[\frac{48}{5} \frac{(\Delta \epsilon / \epsilon) \mu_{0}^{2} \sin ^{3} \mu_{0}}{U^{2} \omega^{4} \epsilon\left(\left\langle J^{2}\right\rangle / \epsilon^{2}\right)}\right]^{1 / 3}
$$

From this, one can compute the RF phase and the energy spread:

$$
\begin{gathered}
\sigma_{E}=\sqrt{\frac{\epsilon}{T_{1}} \tan \frac{\mu_{0}}{2}} \\
\phi=\tan ^{-1} \frac{4 \sin ^{2}\left(\mu_{0} / 2\right)}{T_{1} U \omega}
\end{gathered}
$$

For a Gaussian distribution, these parameters are computed in Table VI.

\section{DROPLET ARC DESIGN II}

$T_{1}$ does not uniquely determine the arc design: it depends on two parameters, $n_{o}$ and $L$. One of these two much be chosen in some rational fashion. To make the match from the linac to the arc perform optimally, it is best to have the beta functions at the end of the linac be similar to those at the end of the arc. For a thin lens FODO cell with a full cell length $L$, the geometric mean of the beta functions at the quadrupoles is

$$
\sqrt{\beta_{D} \beta_{F}}=\frac{L}{2} \csc \frac{\mu_{\perp}}{2}
$$

TABLE VII. Droplet arc parameters when the linac and arc have the same geometric average of their beta functions at the points where they meet.

\begin{tabular}{lrrrr}
\hline \hline$\epsilon(\mathrm{mm})$ & 1.5 & 1.5 & 70 & 70 \\
$\omega / 2 \pi(\mathrm{MHz})$ & 325 & 650 & 325 & 650 \\
Linac passes & 9 & 3 & 9 & 3 \\
\hline$\mu_{\perp}$ end (deg.) & 51 & 23 & 38 & 10 \\
Cells per cavity & 2 & 5 & 2 & 2 \\
Linac cell length (m) & 6.46 & 5.53 & 6.46 & 4.15 \\
$n_{o}$ & 0 & 6 & 7 & 46 \\
Decay loss (\%) & 8.8 & 5.3 & 17.4 & 20.7 \\
$L$, first arc (m) & 11.2 & 20.1 & 15.5 & 34.4 \\
Total arc length $(\mathrm{km})$ & 4.3 & 3.3 & 24.0 & 32.5 \\
\hline \hline
\end{tabular}

where $\mu_{\perp}$ is the betatron phase advance per cell. I will first describe designs where this geometric mean is the same at the end of the first linac pass and for the first droplet arc.

$\sin \left(\mu_{\perp} / 2\right)$ is proportional to the particle momentum for a given cell length and magnetic field gradients in the quadrupoles. To take the most extreme parameters, assume that betatron oscillations become unstable for particles with energy $E_{0}-k \sigma_{E}$, where $E_{0}$ is the linac injection energy and $k$ is the "physical aperture" factor from Table I. Then the phase advance $\mu_{\perp}$ at the end of the linac is given by

$$
\sin \frac{\mu_{\perp}}{2}=\frac{E_{0}-k \sigma_{E}}{E_{0}+U} .
$$

Therefore the ratio of the linac cell length to the arc cell length to keep the geometric product of the beta functions the same is about

$$
\frac{1}{\sqrt{2}} \frac{E_{0}+U}{E_{0}-k \sigma_{E}} .
$$

This takes into account the $\pi / 2$ phase advance in the arc cells.

For a given $T_{1}$, the number of cells increases as the arc cell length increases. Therefore the total arc length and muon decays increase with that cell length. If the geometric mean of the beta functions is kept the same for the linac and the arc, this means the linac cell length should be shorter to have the best performance and lowest cost. I therefore use superconducting quadrupoles for focusing, with a maximum field of $4 \mathrm{~T}$ at $4.5 \sigma$ for a beam at the design energy and $25 \mathrm{~cm}$ of space on either side of the quadrupoles. There will be one cavity between each pair of quadrupoles, with the number of cells (less than or equal to the maximum in Table II) chosen to give the lowest amount of muon decay. The cell length of the linac should be an integer number of RF wavelengths so the RF phasing works for both muon signs.

The resulting designs are given in Table VII. Designs can be constructed that have reasonable performance for the $1.5 \mathrm{~mm}$ longitudinal emittance, but the results are unacceptable for the $70 \mathrm{~mm}$ longitudinal emittance: decay losses and the total arc circumference are too high. For the $325 \mathrm{MHz}$ case, the indivudal arcs are of a moderate length, but the large number of 
TABLE VIII. Droplet arc parameters for a beam with a $70 \mathrm{~mm}$ longitudinal emittance with no beta function matching requirement between the arc and linac.

\begin{tabular}{lrrrr}
\hline \hline$\omega / 2 \pi(\mathrm{MHz})$ & 325 & 325 & 650 & 650 \\
Linac passes & 9 & 9 & 3 & 3 \\
Arc dipole field (T) & 1.5 & 6.0 & 1.5 & 6.0 \\
\hline$n_{o}$ & 11 & 5 & 28 & 15 \\
$L$, first arc (m) & 5.43 & 3.57 & 5.34 & 3.44 \\
Total arc length (km) & 12.0 & 4.3 & 3.2 & 1.2 \\
Decay loss $(\%)$ & 12.3 & 9.1 & 6.2 & 5.3 \\
\hline \hline
\end{tabular}

them leads to an excessive circumference. For the $650 \mathrm{MHz}$ case, the very large energy spread leads to a very low betatron phase advance at the end of the first linac pass, leading to a very long cell length and large number of cells in the droplets arcs. Because of this, despite the smaller number of arcs, the $650 \mathrm{MHz}$ design looks significantly worse than the $325 \mathrm{MHz}$ design.

Therefore, to accelerate the $70 \mathrm{~mm}$ longitudinal emittance with tolerable emittance growth, one will have to accept a beta mismatch between the droplet arc cells and the adjacent linac cells. This will require beta matching in the cells at the end of the droplet, which may have some impact on the performance, since it will be difficult to have the beta match be correct over the entire energy spread in the beam. I will fix the maximum fields in the arc magnets, considering warm (maximum dipole fields of $1.5 \mathrm{~T}$, maximum quadrupole field at $4.5 \sigma$ of $1.0 \mathrm{~T}$ ) and superconducting (maximum dipole fields of $6.0 \mathrm{~T}$, maximum quadrupole field at $4.5 \sigma$ of $4.0 \mathrm{~T}$ ) solutions. To find a solution, I adjust $n_{o}$ and $L$, keeping the dipole and quadrupole fields at their maximum values, so that $n_{o}$ takes the smallest value that keeps $T_{1}$ less than the value from Table VI. The results are given in Table VIII.

\section{CONCLUSIONS}

I have been able to find reasonable parameters for a dogbone RLA that will accelerate a muon beam for both the Higgs factory and high energy collider parameters given in Table I. The important longitudinal parameters are given in Table VI, and approximate droplet arc parameters are given in Table VIII. A $650 \mathrm{MHz}$ linac appears to be a better solution, except the large energy spread in the beam is a cause for concern. Superconducting arcs appear to provide a more cost-effective solution due to a significantly smaller number of magnets and total arc length. However, the larger beta mismatch between the linacs and the arcs and a desner switchyard may lead to a preference for a warm magnet solution. A $325 \mathrm{MHz}$ solution with superconducting magnets is also acceptable, though switchyard crowding may become a significant problem for that case.

If one only wishes to accelerate the beam for the Higgs factory in this machine, then the solutions from Table VII would probably perform better since there is minimal betatron mismatch between the arc and linac, and the magnet density at the switchyard should be low. The $650 \mathrm{MHz}$ solution would be preferable in this case.

To reduce the energy spread for the collider emittance, one could use a racetrack geometry instead of the dogbone geometry. The racetrack geomtry has the additional advantage of treating both charges equally with respect to beam loading. I also expct that two accelerating stages instead of just one would yield better designs.
[1] J.-P. Delahaye et al., Enabling Intensity and Energy Frontier Science with a Muon Accelerator Facility in the U. S.: A White Paper Submitted to the 2013 U. S. Community Summer Study of the Division of Particles and Fields of the American Physical Society, Tech. Rep. FERMILAB-CONF-13-307-APC (Fermilab, 2014) arXiv:1308.0494 [physics.acc-ph].

[2] J. Plouin, G. Devanz, and S. Chel, "Optimized RF design of 704 $\mathrm{MHz}$ beta=1 cavity for pulsed proton drivers," in Proceedings of SRF2011, Chicago, IL, USA (2011) pp. 157-161.

[3] Rong-Li Geng, "SRF technical status and future R\&D," presentation at LCWS12: International Workshop on Future Linear Colliders, University of Texas at Arlington, USA, 22-26 October 2012 (2012)

[4] Daniel Boussard, "Operational experience with the LEP2 SC cavity system," in Fifth European Particle Accelerator Conference, edited by S. Myers, A. Pacheco, R. Pascual, Ch. Petit-JeanGenaz, and J. Poole (Institute of Physics Publishing, Bristol, UK, 1996) pp. 187-191.

[5] J. Scott Berg, Carol Johnstone, and Don Summers, "Dogbone geometry for recirculating accelerators," in Proceedings of the 2001 Particle Accelerator Conference, Chicago, edited by P. Lucas and S. Webber (IEEE, Piscataway, NJ, 2001) pp. 3323-3325.
[6] Y. Alexahin and E. Gianfelice-Wendt, "A 3-TeV muon collider lattice design," in Proceedings of IPAC2012, New Orleans, Louisiana, USA (IEEE, 2012) pp. 1254-1256.

[7] S. Ozaki, R. B. Palmer, M. S. Zisman, and J. C. Gallardo, Feasibility Study-II of a Muon-Based Neutrino Source, Tech. Rep. BNL-52623 (Brookhaven National Laboratory, 2001) http: //www .osti.gov/scitech/servlets/purl/914482.

[8] John Carwardine, Chris Adolphsen, Maura Barone, Barry Barish, Karsten Buesser, Phil Burrows, Jeffrey Clark, Hèlène Mainaud Durand, Gerry Dugan, Eckhard Elsen, Atsuto Enomoto, Brian Foster, Shigeki Fukuda, Wei Gai, Martin Gastal, Rongli Geng, Camille Ginsburg, Susanna Guiducci, Mike Harrison, Hitoshi Hayano, Keith Kershaw, Kiyoshi Kubo, Vic Kuchler, Benno List, Wanming Liu, Shinichiro Michizono, Christopher Nantista, John Osborne, Mark Palmer, James McEwan Paterson, Thomas Peterson, Nan Phinney, Paolo Pierini, Marc Ross, David Rubin, Andrei Seryi, John Sheppard, Nikolay Solyak, Steinar Stapnes, Toshiaki Tauchi, Nobu Toge, Nicholas Walker, Akira Yamamoto, and Kaoru Yokoya, "The international linear collider, technical design report, volume 3.ii: Accelerator baseline design," Reports ANL-HEP-TR-13-20, BNL-100603-2013-IR, IRFU-13-59, CERN-ATS-2013-037, Cockcroft-13-10, CLNS 13/2085, DESY 13-062, FERMILAB 
TM-2554, IHEP-AC-ILC-2013-001, ILC-REPORT-2013-040, INFN-13-04/LNF, JAI-2013-001, JINR E9-2013-35, JLABR-2013-01, KEK Report 2013-1, KNU/CHEP-ILC-20131, LLNL-TR-635539, SLAC-R-1004, ILC-HiGrade-Report2013-003 (2013), http: //www . linearcollider.org/ILC/ Publications/Technical-Design-Report.
[9] S. A. Bogacz, "Low energy stages - 'dogbone' muon RLA," Nucl. Phys. B (Proc. Suppl.) 149, 309-312 (2005).

[10] Alex J. Dragt and Etienne Forest, "Computation of nonlinear behavior of hamiltonian systems using lie algebraic methods," J. Math. Phys. 24, 2734-2744 (1983). 